\title{
Processing Tissues in the Conventional Manner Utilizing Chemicals
}

José A. Mascorro

Department of Structural and Cellular Biology

Tulane University Health Sciences Center

New Orleans, LA 70112

The fine structural detail of diverse biological tissues, and their individual cellular components, has been studied extensively since the advent of the electron microscope. Electron microscopic studies initially subject the desired tissue to treatment with a chemical agent in order to immobilize the living specimen in a way that will eliminate, or minimize, changes in ultrastructural detail. Chemicals such as paraformaldehyde, glutaraldehyde, and osmium tetroxide have become the norm for this first step in the overall procedure for preparing tissues for subsequent study with the electron microscope. Tissues also are treated with alcohols for dehydration and liquid epoxy resins for infiltration and embedding. Infiltrated tissues finally are subjected to gentle heat for a period of several hours in order to produce a hardened block suitable for ultrathin sectioning and staining with solutions of heavy metals. This microscopist has processed biological tissues according to this very conventional, very traditional method for decades. The following information summarizes the process, including personal preferences and opinions based upon many years of experience.

Fixation and Dehydration: Soft biological tissues first are fixed by vascular perfusion utilizing a solution of $3 \%$ glutaraldehyde adjusted to $\mathrm{pH} 7.2$ - 7.3 in $0.2 \mathrm{M}$ phosphate buffer. Fixation also can be performed by the simpler method of injecting or dripping fixative onto the specimen, followed by excision and immersion fixation. On the other hand, perfusion utilizes the arteriolar and terminal capillary beds to rapidly deliver the chemical to the individual cells and has always been the preferred method here. The vascular perfusion proceeds for 20 minutes, after which the desired tissue is dissected out, diced into small $1 \mathrm{~mm}$ cubes while immersed in droplets of fixative, and further fixed in fresh fixative for 20 minutes. The tissues then are rinsed briefly, $2 \times 5$ minutes with occasional swirling, in the buffer vehicle. Secondary fixation is performed with osmium tetroxide, similarly buffered to $\mathrm{pH}$ 7-2 - 7.3 with phosphate, for 30 minutes. As a matter of preference and experience with many tissues, the entire fixation process always is performed with ice-cold fixatives. Presumably, this arrests the extraction of cell components. Prior to dehydration, the specimens again are rinsed rapidly in the buffer vehicle to remove free aldehyde molecules. Dehydration is achieved by passing the specimens through a $50 \%, 70 \%$, $95 \%$, and $100 \%$ graded series of cold absolute ethanol alcohol, 5 - 10 minutes at each step, with swirling. The specimens remain, and are allowed to equilibrate to room temperature, in the $100 \%$ ethanol for 30 minutes with several changes. The use of propylene oxide (PO) as a transitional solvent was deemed unnecessary and abandoned from this technique many years ago [1]. For several reasons, PO is not desirable for this microscopist, and since epoxy resins are sufficiently miscible with ethanol, its use is contraindicated in this opinion.

Infiltration, Embedding, and Polymerization: Epoxy resins are the embedding medium of choice for the preservation of tissues to be sectioned for examination by transmission electron microscopy. Several Epon-like epoxy resins [2] are available to the microscopist today, and 
many infiltration schedules can be used depending upon the tissue type and final viscosity of the chosen embedding medium. In the present technique, tissues are transferred from the last dehydration step to a 1:1 mixture of embedding medium/ethanol for $\sim 15$ minutes, or until the specimens settle to the bottom of the filled vial. This mixture is replaced by a $2: 1$ mix of embedding medium/ethanol for 15-30 minutes, followed by final infiltration in the medium for 3 hours. Vials containing the tissue and infiltrating medium are mounted on a multipurpose rotator at all stages to produce constant agitation and promote optimal infiltration. All epoxy-based embedding medium are relatively viscous, even those designed specifically with a low viscosity character [3]. It seems doubtful that significant infiltration will continue to occur after the fully prepared medium has set for more than 1-2 hours. Therefore, the embedding medium of choice always is prepared while the material is in the last stage of dehydration so as to ensure that it is at its lowest viscosity and capable of optimum penetration. As a matter of convenience, this entire protocol is planned so that infiltration is completed by the end of the working day. The specimens finally are placed in oven-dried Beem capsules and polymerized at $60^{\circ} \mathrm{C}$ for an overnight period of 15 hours. After allowing a few minutes for cooling, the polymerized tissue blocks are sectioned and the sections then treated with appropriate lead citrate and uranyl acetate stains subsequent to microscopic study.

It is correct to say that one's tissue dictates one's protocol. This present technique for fixing, dehydrating, infiltrating, and embedding a wide range of biological material has proven successful for producing tissues that can be utilized both for research and teaching purposes. It seems to work well with most body organ tissues. In particular, the simple and straightforward methods described here seem quite appropriate for the study of peripheral, autonomic nervous tissue which has been a focus for this investigator. This protocol is not presented as a panacea, nor is it intended to supplant other techniques. However, it represents 41 years worth of successful experience in the TEM laboratory and can be recommended without reservations.

\section{References:}

[1] Hilton Mollenhauer, via personal communication.

[2] J.A. Mascorro and G.S. Kirby, Proceedings of XII ICEM. 3 (1990) 138.

[3] J.A. Mascorro and G.S. Kirby, Micros Res and Tech. 20 (1992) 105. 